\title{
Intravenous Tranexamic Acid Decreases Blood Transfusion in Off-Pump Coronary Artery Bypass Surgery: A Meta-analysis
}

\author{
Liang Sun MD, ${ }^{1,2}$ Haiyan An, MD,${ }^{1}$ Yi Feng, $M D^{1}$ \\ ${ }^{1}$ Department of Anesthesiology, Peking University People's Hospital, Beijing, China; ${ }^{2}$ Department of Anesthesiology and \\ Critical Care, Perelman School of Medicine, University of Pennsylvania, Philadelphia, PA, USA
}

\section{ABSTRACT}

Background: Tranexamic acid (TXA) has been widely used during on-pump coronary artery bypass graft (CABG) surgery owing to its antifibrinolytic effect. However, the efficacy and safety of TXA in off-pump CABG surgery remains unconfirmed, especially intravenous (IV) administration.

Objective: The aim of this study was to evaluate the effectiveness and safety of IV administration of TXA in off-pump CABG settings.

Methods and Results: A comprehensive literature search was performed to identify randomized controlled trials (RCTs) that compared IV use of TXA with placebo in the reduction of postoperative 24-hour blood transfusion, as well as postoperative death and thrombotic events. The combined estimations were compiled with a fixed-effects model or, if heterogeneity existed, a random-effects model. Funnel plots and Egger's test were used to assess potential publication bias. Subgroup analyses were used to explore possible sources of heterogeneity. In total, 12 RCTs met the inclusion criteria. IV administration of TXA significantly reduced the risk of packed red blood cell (PRBC) transfusion [risk ratio $(\mathrm{RR})=0.61,95 \%$ confidence interval $(\mathrm{CI})$ 0.503 to $\left.0.756, P<.001, \mathrm{I}^{2}=0.0 \%\right)$ during the 24 hours after surgery. However, there was no statistical significance in platelet $\left(\mathrm{RR}=0.613,95 \% \mathrm{CI} 0.112\right.$ to $\left.3.348, P=.572, \mathrm{I}^{2}=0.0 \%\right)$ or total fresh frozen plasma $(\mathrm{FFP})(\mathrm{RR}=0.511,95 \% \mathrm{CI} 0.246$ to $\left.1.063, P=.073, \mathrm{I}^{2}=0.0 \%\right)$ transfusion. Also, no significant difference was found in major adverse events (death or thrombotic complications $)\left(\mathrm{RR}=0.917,95 \% \mathrm{CI} 0.532\right.$ to $1.581, P=.756, \mathrm{I}^{2}$ $=0.0 \%$ ) between the 2 groups. Interestingly, further subgroup analysis demonstrated that IV TXA decreased the risk of prothrombin time (PT)- and international normalized ratio (INR)guided FFP transfusion $(\mathrm{RR}=0.462,95 \%$ CI 0.296 to 0.721 , $\left.P=.001, \mathrm{I}^{2}=0.0 \%\right)$.

Conclusion: IV TXA was effective in reducing allogeneic blood component transfusion (PRBCs and PT- or INRguided FFP transfusion), without increasing the incidence of postoperative death or thrombotic complications in off-pump CAB surgery.

Received October 30, 2019; received in revised form December 6, 2019; accepted December 9, 2019.

Correspondence: Department of Anesthesiology, Peking University People's Hospital. No.11, Xi Zhi Men Nan Da fie, Xicheng District, Beïing 100044, China; 86-108832-5590; fax 86-10-8832-5590; (e-mail: yifeng65@outlook.com).

\section{INTRODUCTION}

To date, postoperative hemorrhage remains a major concern in the setting of cardiac surgery, which includes coronary artery bypass grafting (CABG) [Knapik 2019]. Excessive bleeding increases the requirement for allogeneic blood transfusion, which not only exposes patients to the risks of blood-borne disease and hemolytic reactions, but also poses a socioeconomic burden [Trevisan 2016; Zhou 2017]. Theoretically, since fibrinolysis has a crucial role in bleeding after cardiac surgery [Faraoni 2018], administration of fibrinolysis inhibitors is a promising strategy to reduce the risk of postoperative blood loss in patients undergoing CABG surgery, especially on-pump CABG.

Tranexamic acid (TXA) exerts its antifibrinolytic effect by blocking the lysine-binding sites on plasminogen molecules, increasing the clotting potential of blood, and thereby reducing blood loss [Xu 2019]. Previously published evidence

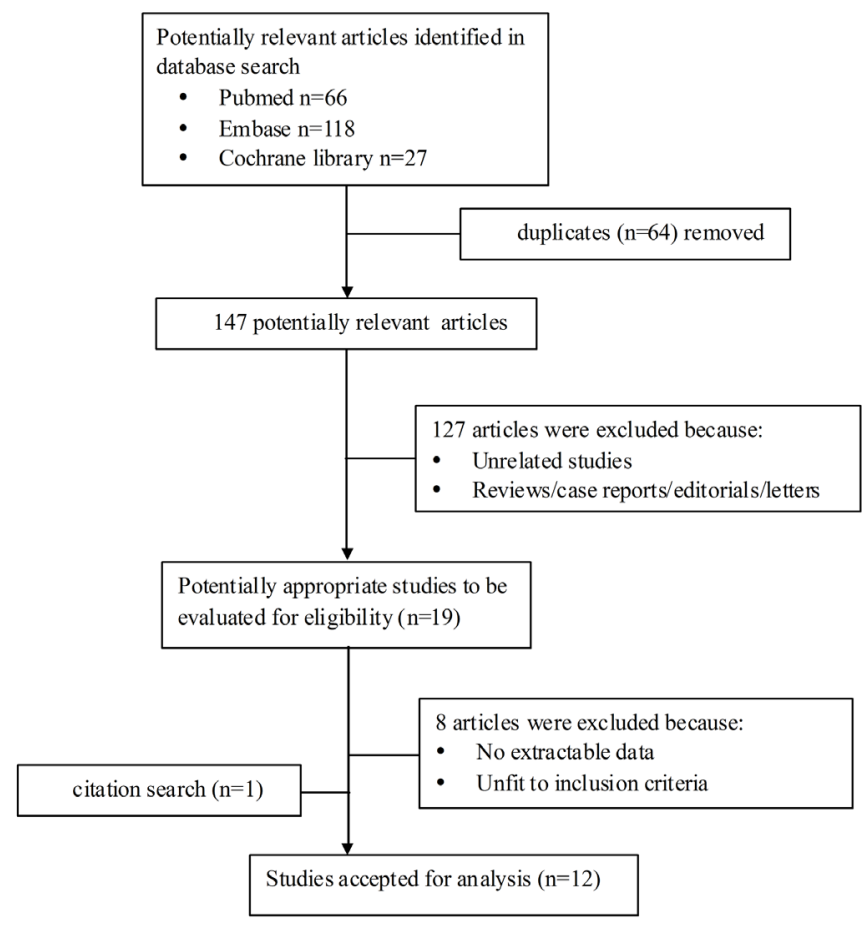

Figure 1. Flow chart of meta-analysis. 
Table 1. Individual RCT Methodological Quality

\begin{tabular}{|c|c|c|c|c|c|c|c|}
\hline Myles 2017 & Yes & Yes & Yes & Yes & Yes & Yes & Yes \\
\hline Ahn 2012 & Yes & Yes & Yes & Yes & Yes & Yes & Yes \\
\hline Taghaddomi 2009 & Yes & Yes & Yes & Yes & Yes & Yes & Yes \\
\hline Mehr-Aein 2007 & $?$ & Yes & Yes & Yes & Yes & Yes & Yes \\
\hline Murphy 2006 & Yes & Yes & Yes & Yes & Yes & Yes & Yes \\
\hline Wei 2006 & $?$ & $?$ & $?$ & $?$ & Yes & Yes & Yes \\
\hline Casati 2001 & $?$ & Yes & Yes & Yes & Yes & Yes & Yes \\
\hline
\end{tabular}

Yes, low risk of bias; no, high risk of bias; ?, unclear risk of bias.

substantiates that TXA reduces blood loss and transfusions associated with on-pump CABG surgery [Andreasen 2004; Shi 2013; Zhang 2018]. Since in the 1990s, off-pump CAB, with its advantages of less bleeding and injury, has emerged as an established alternative surgical technique [Adler 2011]. Recently, a meta-analysis was performed to elucidate the blood-saving efficacy and safety of TXA during off-pump $\mathrm{CAB}$ surgery. However, the authors enrolled the studies investigating both the intrapleural (IP) and intravenous (IV) use of TXA in reducing blood transfusion, and thus the efficacy of IV TXA was still equivocal in off-pump CAB scenarios [Dai 2018]. Meanwhile, the safety profile of TXA may be different to some extent in off-pump CAB compared with an onpump setting. Particularly, activation of higher-than-normal levels of fibrinogen might promote a higher risk of adverse thrombotic complications in off-pump CAB surgery [Casati 2001]. Accordingly, taking into account the preference of the IV route in clinical settings as well as different pharmacokinetics and pharmacodynamics between IP and IV deliveries, we conducted a meta-analysis of randomized controlled trials (RCTs) to evaluate the effectiveness and safety of TXA in offpump CAB operation, focusing solely on IV administration.

\section{METHODS}

\section{Search Strategy}

We performed a systematic review and meta-analysis according to the Quality of Reporting of Meta-analyses (QUOROM) recommendations for improving the quality of meta-analyses [Moher 1999]. We searched Pubmed, Embase, and the Cochrane Central Register of Controlled Trials up to April 2019 for relevant studies. Search strategies for subject headings and key words included (1) coronary artery bypass, coronary surgery, coronary bypass, myocardial revascularization, coronary revascularization, coronary artery surgery, or CABG; (2) off-pump, beating heart, or off pump; and (3) tranexamic acid, antifibrinolytic, fibrinolysis inhibitor, TXA, Cyklokapron, Lysteda, AMCHA (4-aminomethylcyclohexancarbonic acid), Transamin, or Exacyl. No restrictions were imposed. A secondary reference review was conducted.

\section{Eligibility Criteria}

Titles, abstracts, and full texts were reviewed. Eligible studies met the following criteria: (1) RCT that involved patients undergoing off-pump CABG; (2) patients received IV TXA or placebo perioperatively; and (3) article published in English.

\section{Data Extraction}

We extracted the following information from each study: first author, year of publication, country, patient age and weight, preoperative cardiovascular complications, number of grafts, TXA (route of administration and dose), antiplatelet drugs before surgery, transfusion indications [packed red blood cells (PRBCs), fresh frozen plasma (FFP), platelets], and intraoperative use of cell salvage. Independent investigators calculated and tabulated the data with a standard extraction formula. Discrepancies were discussed by 2 authors.

\section{Quality Assessment}

The methodological quality of included studies was evaluated using the Cochrane Collaboration's tool for assessing risk of bias in randomized trials, and disagreements were resolved through discussion (Table 1) [Higgins 2011]. The tool consists of 7 items describing random sequence generation, 
Table 2. Characteristics of Included Studies

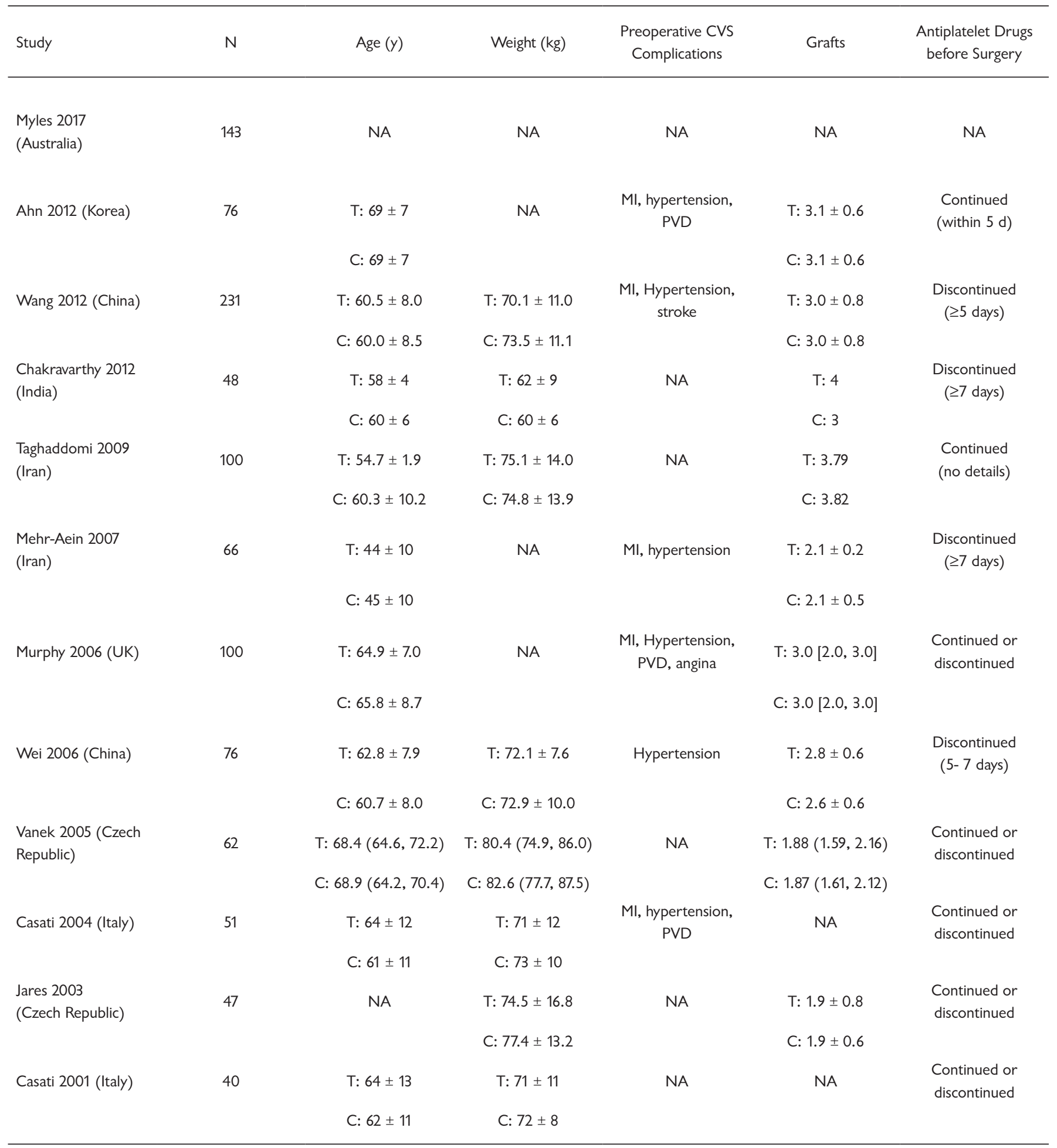

Data are presented as mean \pm SD, median [IQR], or mean (95\% confidence interval). BSI, before skin incision; C, control; CVS, cardiovascular system; FFP, fresh frozen plasma; HCT, hematocrit; Hb, hemoglobin; INR, international normalized ratio; IV, intravenous; L, loading dose; M, maintenance dose; MI, myocardial infarction; NA, not available; PLT, platelet; PRBC, packed red blood cell; PT, prothrombin time; PVD, peripheral vascular disease; T: test; TXA, tranexamic acid. 
Table 2. Characteristics of Included Studies [Cont.]

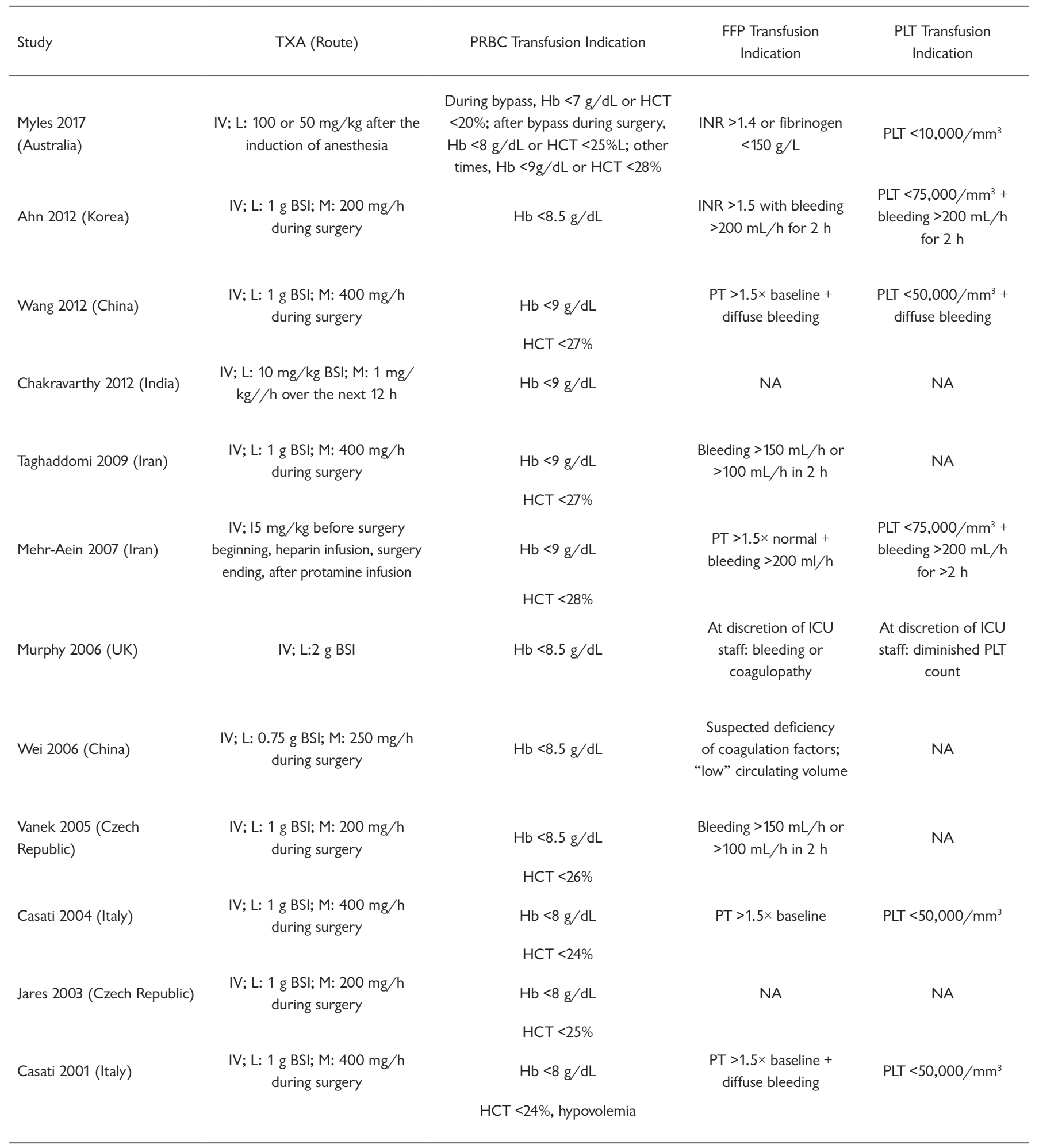




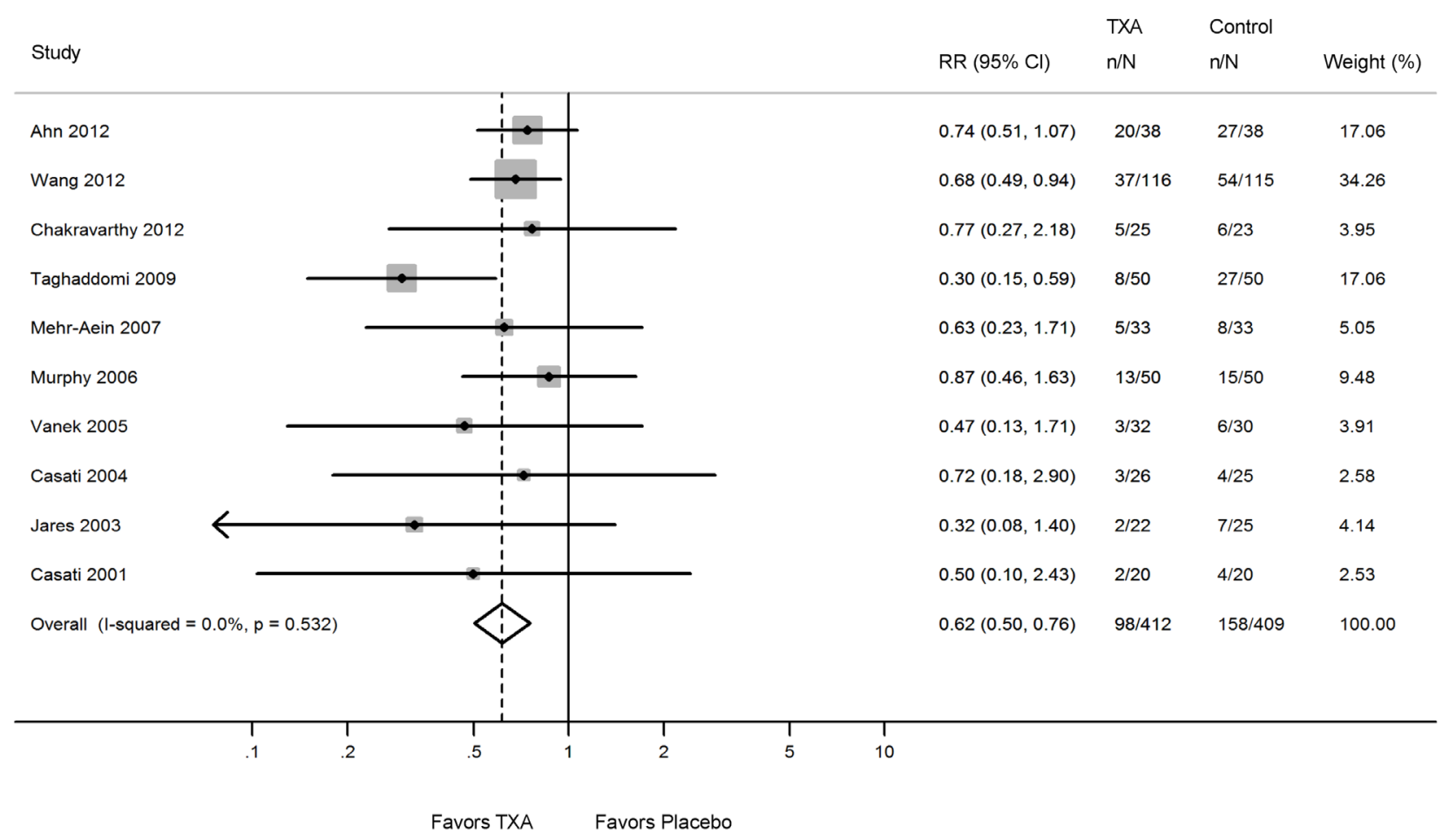

Figure 2. Forest plot of the meta-analysis of tranexamic acid (TXA) on packed red blood cell (PRBC) transfusion. Cl, confidence interval; RR, risk ratio.

allocation concealment, blinding of participants and personnel, blinding of outcome assessment, incomplete outcome data, selective reporting, and other biases. For each item, we assigned a judgment of high, low, or unclear risk of material bias [Higgins 2008].

\section{Postoperative Outcome Measurements}

The primary endpoint was allogeneic blood component transfusion (PRBCs, FFP, and platelets) during the first 24 hours of postoperative care. The secondary endpoint was a composite of postoperative death and thrombotic events (myocardial infarction, stroke, pulmonary embolism or deep vein thrombosis, and renal insufficiency) [Myles 2017].

\section{Statistical Analyses}

We pooled data across studies and calculated the risk ratio (RR) and associated 95\% confidence interval (CI) for each dichotomous outcome using a fixed-effects model or, if heterogeneity existed, a random-effects model. Heterogeneity across studies was tested by using the $\mathrm{I}^{2}$ statistic, which is a quantitative measure of inconsistency across studies. $P<.10$ and $\mathrm{I}^{2}>50 \%$ indicated significant heterogeneity and prevented reliance on a combination of the study results.

Subgroup analyses were conducted to explore possible explanations for potential sources of heterogeneity. Sensitivity analyses were used to assess the robustness of our results by removing each study at a time to obtain and evaluate the remaining overall estimates. The possibility of publication bias was assessed by using the visual inspection of Begg funnel plots (if the number of included studies was more than 10) and Egger's test [Begg 1994; Egger 1997]. A significant publication bias was defined as $P<.1$. Trim-and-fill computation was used to estimate the effect of publication bias on the interpretation of the results [Mavridis 2014]. All analyses were performed using STATA version 11.2 (Stata Corp LP, College Station, TX). A $P$ value $<.05$ was considered statistically significant.

\section{RESULTS}

\section{Literature Search and Study Characteristics}

We initially retrieved 211 articles from PubMed and Embase databases and the Cochrane Central Register of Controlled Trials. Most of these references were excluded according to the previous criteria. In the end, 12 independent studies were included in our meta-analysis [Casati 2001; Jares 2003; Casati 2004; Vanek 2005; Murphy 2006; Wei 2006; Mehr-Aein 2007; Taghaddomi 2009; Ahn 2012; Chakravarthy 2012; Wang 2012; Myles 2017]. The detailed steps of our literature search and study selection are described in Figure 1.

The characteristics of the 12 RCTs are presented in Table 2. Regarding methodological quality, 7 studies were shown to have low risk of bias [Casati 2004; Vanek 2005; Murphy 2006; Taghaddomi 2009; Ahn 2012; Wang 2012; Myles 2017], 4 fell into the moderate-risk category [Casati 2001; Wei 2006; 


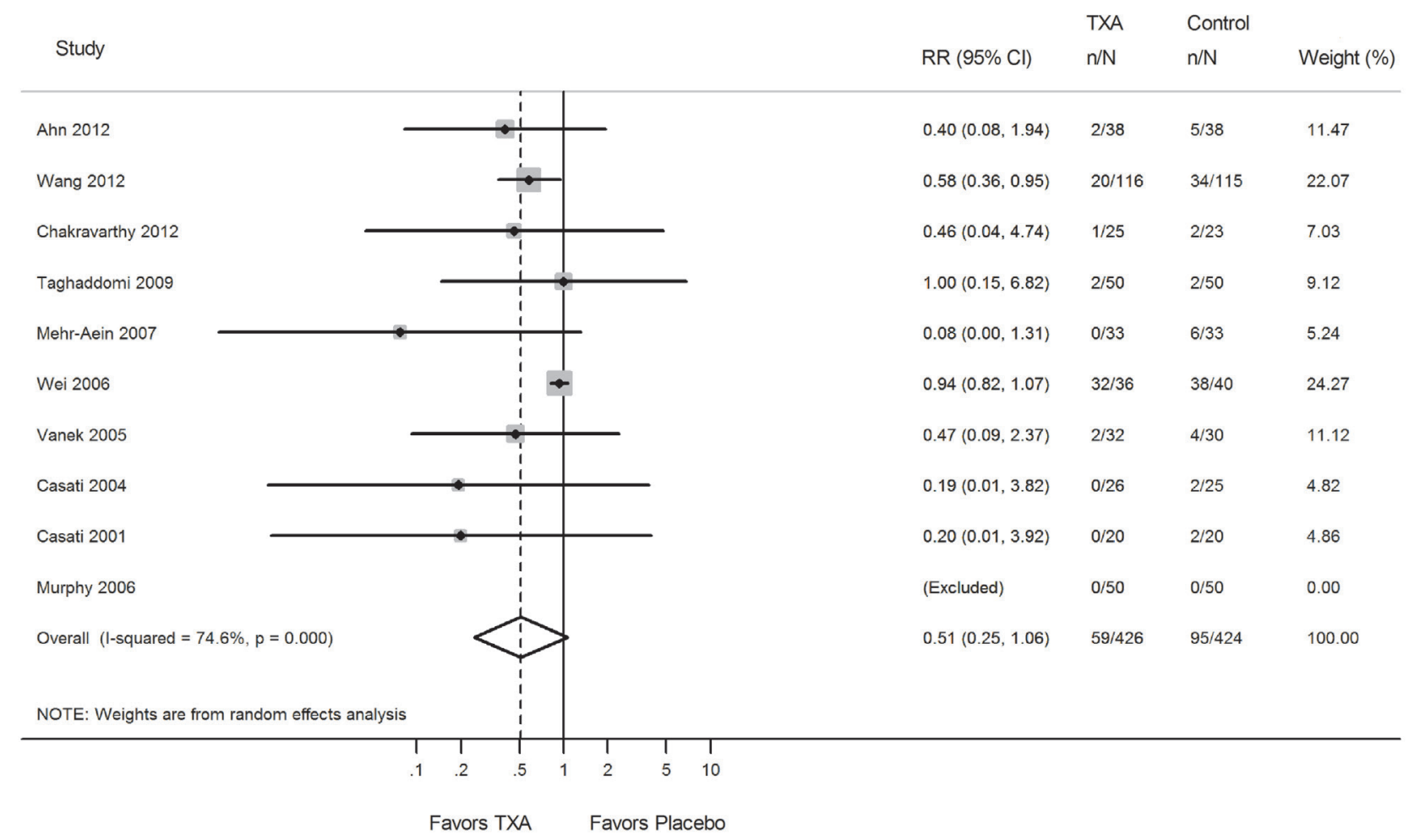

Figure 3. Forest plot of the meta-analysis of tranexamic acid (TXA) on fresh frozen plasma (FFP) transfusion. Cl, confidence interval; RR, risk ratio.

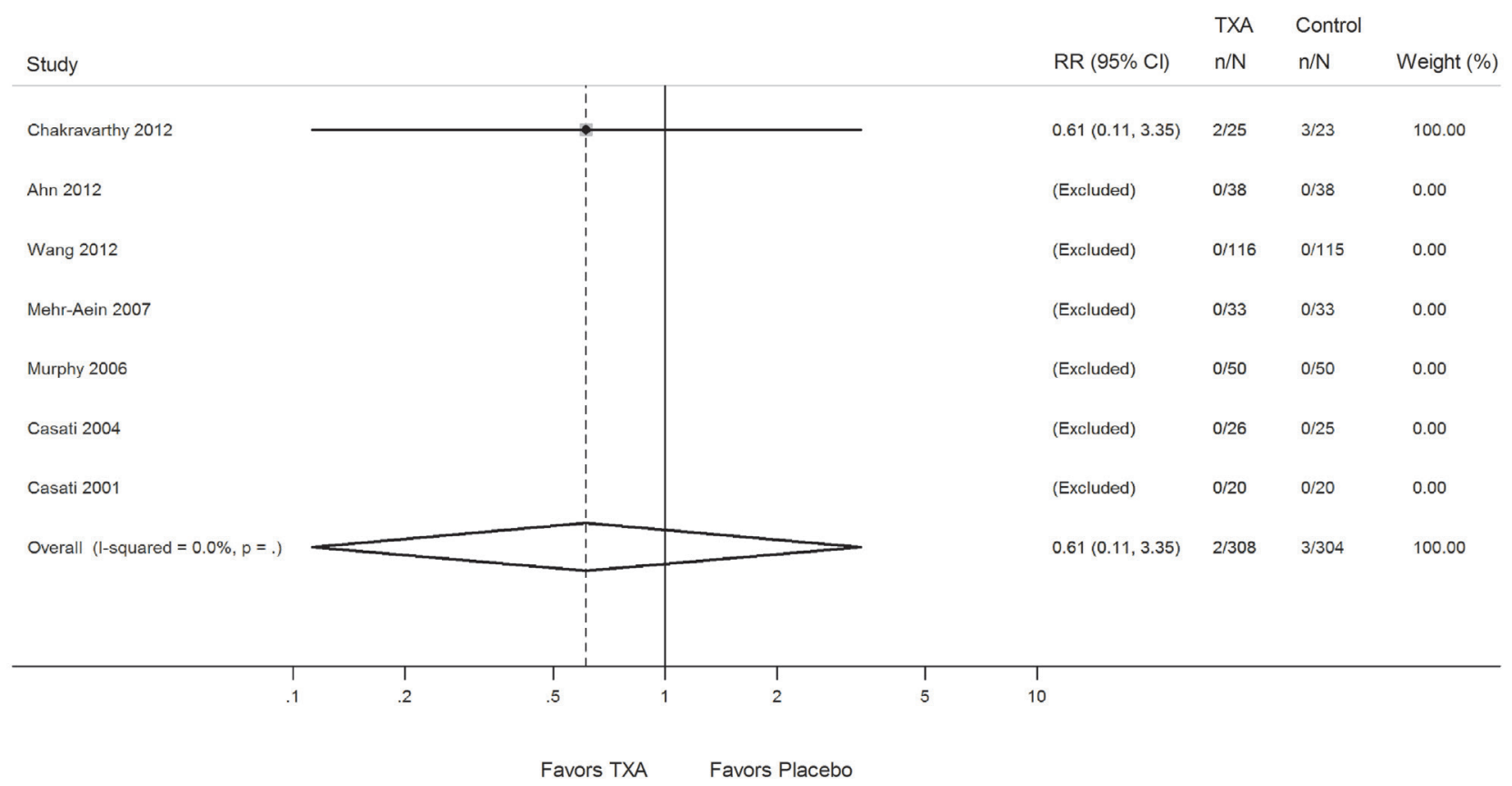

Figure 4. Forest plot of the meta-analysis of tranexamic acid (TXA) on platelet transfusion. Cl, confidence interval; RR, risk ratio. 


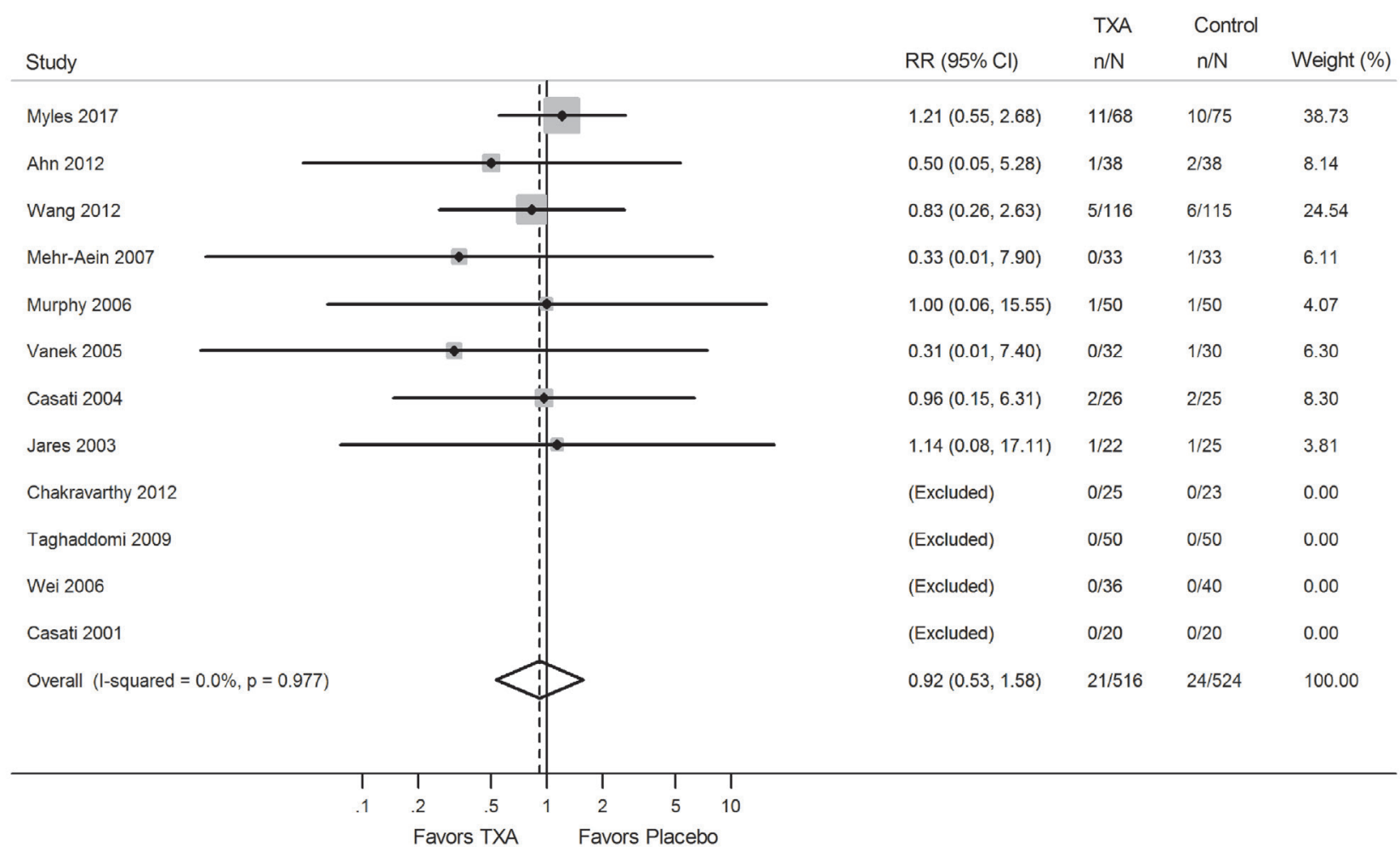

Figure 5. Forest plot of the meta-analysis of tranexamic acid (TXA) on postoperative death and thrombotic events. Cl, confidence interval; RR, risk ratio.

Mehr-Aein 2007; Chakravarthy 2012], and only 1 study was at high risk [Jares 2003]. The meta-analysis included 12 articles (516 patients in the TXA group and 524 in the placebo group). Among these studies, 10 reported the risk of PRBC transfusion [Casati 2001; Jares 2003; Casati 2004; Vanek 2005; Murphy 2006; Mehr-Aein 2007; Taghaddomi 2009; Ahn 2012; Chakravarthy 2012; Wang 2012], 10 focused on the risk of FFP transfusion [Casati 2001; Casati 2004; Vanek 2005; Murphy 2006; Wei 2006; Mehr-Aein 2007; Taghaddomi 2009; Ahn 2012; Chakravarthy 2012; Wang 2012], 7 showed the risk of platelet transfusion [Casati 2001; Casati 2004; Murphy 2006; Mehr-Aein 2007; Ahn 2012; Chakravarthy 2012; Wang 2012], and all 12 included the risk of postoperative death as well as thrombotic complications [Casati 2001; Jares 2003; Casati 2004; Vanek 2005; Murphy 2006; Wei 2006; Mehr-Aein 2007; Taghaddomi 2009; Ahn 2012; Chakravarthy 2012; Wang 2012; Myles 2017].

\section{TXA and the Risk of PRBC Transfusion}

IV TXA was associated with a decreased incidence of PRBC transfusion $(\mathrm{RR}=0.617 ; 95 \% \mathrm{CI} 0.503$ to $0.756, P<$ $.001)$, with no heterogeneity $\left(P=.532, \mathrm{I}^{2}=0 \%\right)$ (Figure 2$)$.

\section{TXA and the Risk of FFP Transfusion}

The pooled analysis of 10 enrolled studies found that IV TXA was not associated with a decreased incidence of FFP transfusion $(\mathrm{RR}=0.511 ; 95 \% \mathrm{CI} 0.246$ to $1.063, P=.073)$, with moderate heterogeneity $\left(P<.001, \mathrm{I}^{2}=74.6 \%\right)$, thus indicating a neutral effect (Figure 3 ).

\section{TXA and the Risk of Platelet Transfusion}

No association was found between IV TXA and the risk of platelet transfusion ( $\mathrm{RR}=0.613 ; 95 \% \mathrm{CI} 0.112$ to 3.348 , $P=.572)$, with no heterogeneity $\left(\mathrm{I}^{2}=0 \%\right)$ (Figure 4$)$.

\section{TXA and the Risk of Postoperative Death and Thrombotic Events}

There was no association between IV TXA and incidence of death and thrombotic events $(\mathrm{RR}=0.917 ; 95 \% \mathrm{CI} 0.532$ to $1.581, P=.756)$, with no heterogeneity $\left(P=.977 ; \mathrm{I}^{2}=74.6 \%\right)$ (Figure 5).

\section{Subgroup and Sensitivity Analyses}

To explore the possible effects of confounding factors on TXA with regard to preventing the risk of FFP transfusion, we performed a subgroup analysis. When the subgroup analysis was restricted to FFP transfusion indications, the heterogeneity disappeared ( $\mathrm{I}^{2}=0 \%$ in each subgroup) when comparing the group using prothrombin time (PT) or international normalized ratio (INR) guidance versus the group without PT or INR guidance, thus indicating that FFP transfusion indications may be the source of heterogeneity. Intriguingly, we found that IV TXA significantly reduced the risk of FFP transfusion in the PT and INR group $(\mathrm{RR}=0.462,95 \% \mathrm{CI}$ 


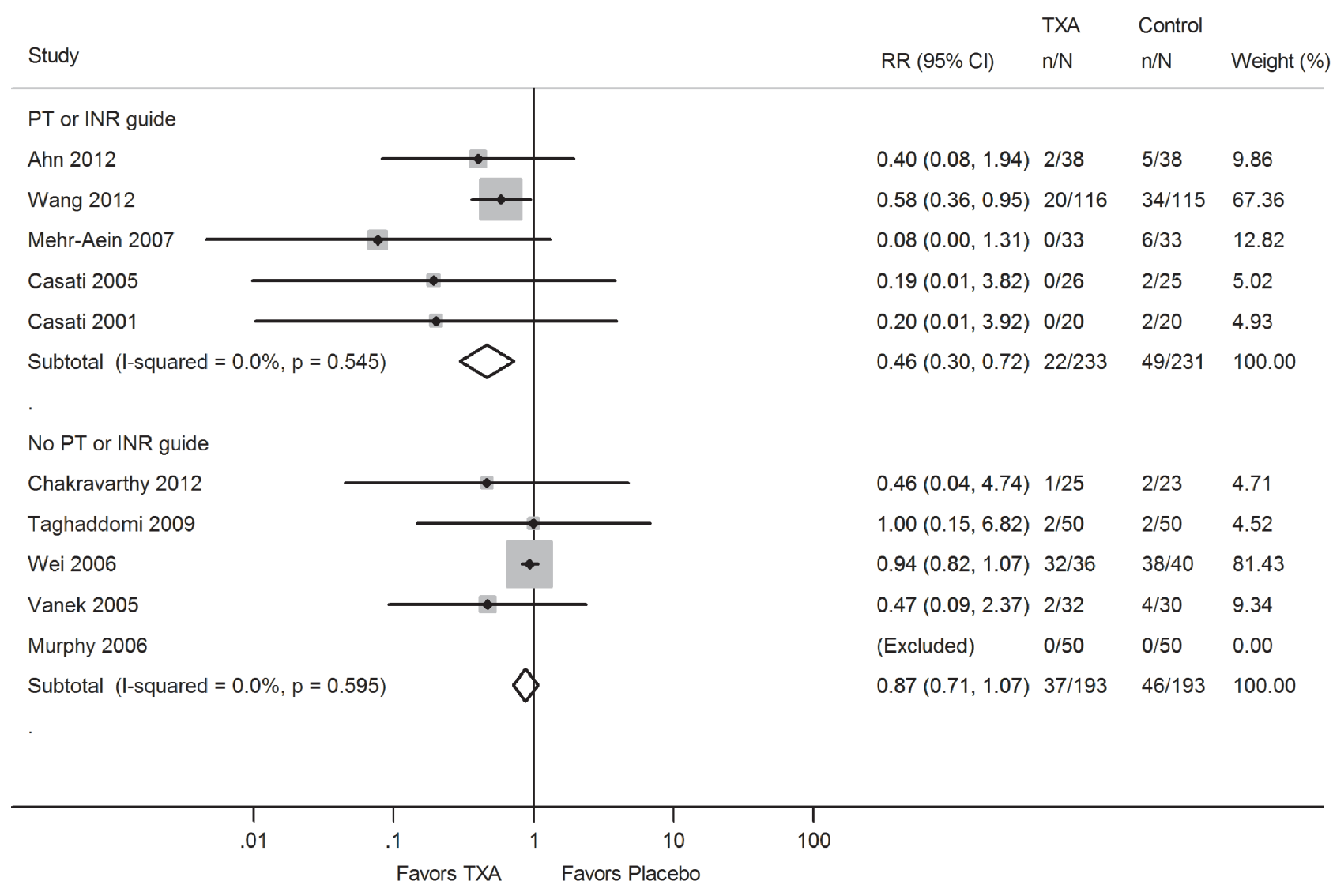

Figure 6. Forest plot of the meta-analysis of tranexamic acid (TXA) on fresh frozen plasma (FFP) transfusion using subgroup analysis. Cl, confidence interval; INR, international normalized ratio; PT, prothrombin time; RR, risk ratio.

0.296 to $\left.0.721, P=.001, \mathrm{I}^{2}=0.0 \%\right)$. However, in the group without PT and INR guidance, TXA did not decrease the risk of FFP transfusion $\left(\mathrm{RR}=0.873,95 \%\right.$ CI 0.714 to $1.067, \mathrm{I}^{2}=$ $0.0 \%$ ) (Figure 6).

In addition, sensitivity analyses excluding each study at a time revealed that after excluding 3 studies [Murphy 2006; Wei 2006; Taghaddomi 2009], the remaining pooled estimates were not consistent with the overall effect (Figure 7). We found that all FFP transfusions were performed without PT or INR guidance in these studies. Therefore, sensitivity analyses further substantiated that IV TXA can reduce the risk of PT- or INR-guided FFP transfusion.

\section{Publication Bias}

Visual inspection of the funnel plot did not indicate any evidence of obvious and substantial asymmetry for TXA in the risk of PRBC transfusion (Begg's test, $P=.283$; Egger's test, $\mathrm{P}=.292$ ). However, the funnel plot did indicate possible deviations from symmetry for TXA in the risk of FFP transfusion (Begg's test, $P=.917$; Egger's test, $P=.014$ ) and postoperative death and thrombotic events (Begg's test, $P=.266$; Egger's test, $P=.048$ ) (Figure 8). Subsequent trim-and-fill analyses revealed that no trimming was performed and data were unchanged, indicating that the asymmetry did not originate from a publication bias.

\section{DISCUSSION}

CABG surgery is often associated with perioperative bleeding and thus the need for allogeneic blood component transfusion. In terms of bleeding concerns, fibrinolysis is an important factor. In the literature, on-pump CABG surgery is associated with excessive and earlier fibrinolytic activity than off-pump surgery [Velioglu 2019], which may partly explain why off-pump CAB surgery is usually accompanied by less blood loss and fewer allogeneic transfusions in clinical settings [Al-Ruzzeh 2003]. Therefore, the efficacy and safety profile of TXA in off-pump CAB surgery may be quite different from that in an on-pump setting. Our meta-analysis of 12 RCTs showed that, in off-pump CAB surgery, TXA exerted great efficacy in reducing allogeneic blood component transfusion (especially PRBC and PT- or INR-guided FFP transfusion), without increasing the incidence of postoperative death and thrombotic events. Additionally, the current study revealed that the use of TXA approximately 


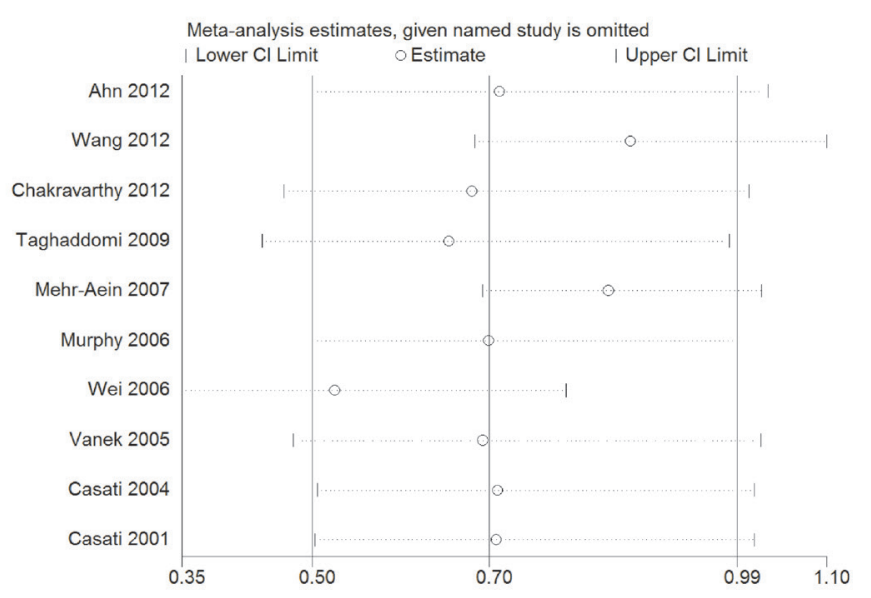

Figure 7. Sensitivity analysis evaluating the influence of each individual study (left side) on the overall estimate. The remaining results with $95 \%$ confidence interval $(\mathrm{Cl})$ are presented.

halved postoperative $\mathrm{PRBC}$ transfusion requirements (RR $=0.617,95 \%$ CI 0.503 to $0.756, P<.001)$. Previous metaanalysis of TXA in on-pump CABG surgery concluded an RR of 0.42 (95\% CI 0.27 to 0.64 ) for PRBC transfusion [Laupacis 1997]. This would suggest that IV administration of TXA is as effective in off-pump CAB surgery as it is in onpump CAB. Recently, another meta-analysis that included IV and IP administration of TXA in off-pump CAB surgery also reported a similar RR, 0.62 (95\% CI 0.50 to 0.75$)$, strengthening our conclusion [Dai 2018].

We did not, however, perform a pooled analysis on postoperative blood loss, for the following reasons. First, it is easier to accept that excessive bleeding is definitely accompanied by allogeneic blood transfusion. Therefore, it is much more meaningful to focus on transfusion requirements. Second, blood loss has typically been proven to be an inconsistent measure of outcomes for meta-analysis. Most studies use median values, which may make a meta-analysis invalid even after data is properly transitioned to mean values, especially taking into account the small size of the identified studies [Deeks 2008].

Differing criteria regarding thresholds for allogeneic blood component transfusion prevent firm conclusions with regard to the risk of FFP transfusion. There is an apparent difference between results within all of the included studies and subgroup analysis (PT or INR guidance or not). Nevertheless, under the guidance of laboratory testing (e.g., PT or INR) in clinical settings, the FFP transfusion strategy is more reasonable and cost efficient. Thus, our conclusion that TXA can reduce the risk of FFP transfusion only in the PT- or INR-guided group is reliable. Our finding of IV TXA administration during FFP transfusion was different from that in a recent meta-analysis [Dai 2018]. That study deduced that TXA has the ability to reduce the incidence of both total and PT-guided FFP transfusion. After analyzing the differences between our study and that meta-analysis, it was obvious that

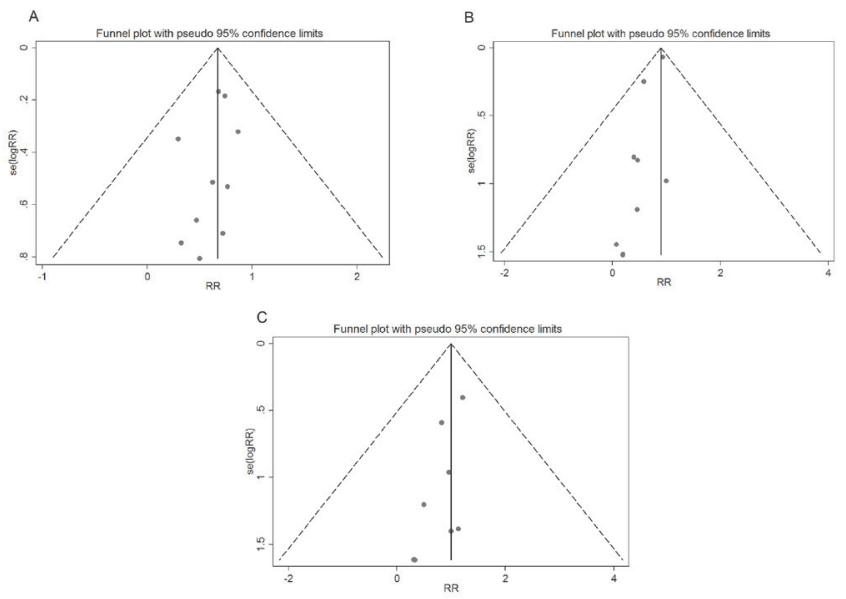

Figure 8. Funnel plot of studies included in the meta-analysis. A, Tranexamic acid (TXA) on packed red blood cell (PRBC) transfusion. $B$, TXA on fresh frozen plasma (FFP) transfusion. C, TXA on postoperative death and thrombotic events. $\mathrm{RR}$, risk ratio.

the criteria for subgroup classification were quite different. Our subgroup analysis of FFP transfusion under a laboratory testing threshold (PT or INR) is more reasonable and reliable in clinical practice.

Seven studies in this meta-analysis reported the risk of platelet transfusion, but most did not report the existence of patients who really needed platelet transfusion. Therefore, our conclusion that TXA did not reduce platelet transfusion requirements still needs to be elucidated in the future.

Although the present results do not substantiate the claim that death or thrombotic events occurred after IV administration of TXA in off-pump CAB surgery, there are limitations on making such concrete conclusions. Assuming 5\% of patients experienced postoperative death and thrombotic events in the control group, to significantly ( $\alpha=0.05$, power $=0.9$ ) detect an increase of $10 \%$ in the TXA group, a sample size of 1156 patients would be required [Schulz 2005]. It is possible that the number of patients in our study (1040) was insufficient to detect these drug-associated significant events. Additionally, most of the retrieved studies monitored death or thrombotic events for only a relatively short period of time. It is possible that these clinically significant events may manifest at a later date [Kurlansky 2003]. Finally, we did not perform a pooled analysis on the incidence of seizures with the use of TXA, because these events were seldom cited in the included studies, although it is worth mentioning that associations between TXA and seizures have been reported previously in cardiac surgery [Zhou 2017]. In 1 study [Myles 2017], 15 patients $(0.7 \%)$ experienced postoperative seizures within the TXA group, but only 2 patients $(0.1 \%)$ in the placebo group $(\mathrm{RR}=7.60,95 \%$ CI 1.80 to $68.70, P=.002)$. The effect of TXA on the incidence of seizures in off-pump CAB settings remains unclear, because that study mixed the on-pump and off-pump CABG cases together.

There are some limitations in our meta-analysis. First, the sample size in some of the included studies was limited. 
Second, only 7 of 12 included trials were designed with low risk of bias; the others were of moderate or high risk. Third, we did not evaluate the efficacy of administrating TXA on postoperative blood loss, which may be a major concern to some. Finally, we did not investigate the possible effect of TXA in the context of its dosing and regimen.

\section{CONCLUSIONS}

In summary, this meta-analysis of the currently available RCTs illustrated the effectiveness of reducing the exposure to PRBC and PT- or INR-guided FFP transfusion by IV administration of TXA during the 24 hours after off-pump CAB surgery. There was no evidence to validate that TXA increases the risk of postoperative mortality or thrombotic complications compared with placebo; however, this study may be insufficiently powered to exclude an association between TXA and adverse events. For future work, a multicenter, well-designed RCT with a large enough sample size will be required to confirm the efficacy and safety of TXA during off-pump CAB surgery.

\section{ACKNOWLEDGMENT}

We thank Denton-Yves Ballough from the Department of Anesthesiology and Critical Care, Perelman School of Medicine, University of Pennsylvania, Philadelphia, for his work on editing this manuscript.

\section{REFERENCES}

Adler MSC, Brindle W, Burton G, et al. 2011. Tranexamic acid is associated with less blood transfusion in off-pump coronary artery bypass graft surgery: A systematic review and meta-analysis. J Cardiothorac Vasc Anesth 25:26-35.

Ahn SW, Shim JK, Youn YN, et al. 2012. Effect of tranexamic acid on transfusion requirement in dual antiplatelet-treated anemic patients undergoing off-pump coronary artery bypass graft surgery. Circ J 76:96-101.

Al-Ruzzeh S, Ambler G, Asimakopoulos G, et al. 2003. Off-pump coronary artery bypass (OPCAB) surgery reduces risk-stratified morbidity and mortality: A United Kingdom Multi-Center Comparative Analysis of Early Clinical Outcome. Circulation 108 (suppl 1):I1-I8.

Andreasen JJ, Nielsen C. 2004. Prophylactic tranexamic acid in elective, primary coronary artery bypass surgery using cardiopulmonary bypass. Eur J Cardiothorac Surg 26:311-7.

Begg CB, Mazumdar M. 1994. Operating characteristics of a rank correlation test for publication bias. Biometrics 50:1088-101.

Casati V, Della VP, Benussi S, et al. 2004. Effects of tranexamic acid on postoperative bleeding and related hematochemical variables in coronary surgery: Comparison between on-pump and off-pump techniques. J Thorac Cardiovasc Surg 128:83-91.

Casati V, Gerli C, Franco A, et al. 2001. Activation of coagulation and fibrinolysis during coronary surgery: On-pump versus off-pump techniques. Anesthesiology 95:1103-9.

Casati V, Gerli C, Franco A, et al. 2001. Tranexamic acid in off-pump coronary surgery: A preliminary, randomized, double-blind, placebocontrolled study. Ann Thorac Surg 72:470-5.

Chakravarthy M, Muniraj G, Patil S, Suryaprakash S, Mitra S, Shivalingappa B. 2012. A randomized prospective analysis of alteration of hemostatic function in patients receiving tranexamic acid and hydroxyethyl starch (130/0.4) undergoing off pump coronary artery bypass surgery. Ann Card Anaesth 15:105-10.

Dai Z, Chu H, Wang S, Liang Y. 2018. The effect of tranexamic acid to reduce blood loss and transfusion on off-pump coronary artery bypass surgery: A systematic review and cumulative meta-analysis. J Clin Anesth 44:23-31.

Deeks JJ, Higgins JPT, Altman DG. 2008. Analysing data and undertaking meta-analyse. In: Higgins JPT, Green S, eds. Cochrane Handbook for Systematic Reviews of Interventions. Chichester, UK: John Wiley \& Sons.

Egger M, Davey SG, Schneider M, Minder C. 1997. Bias in meta-analysis detected by a simple, graphical test. BMJ 315:629-34.

Faraoni D, Rahe C, Cybulski KA. 2018. Use of antifibrinolytics in pediatric cardiac surgery: Where are we now? Paediatr Anaesth 29:435-40.

Higgins JP, Altman DG, Gotzsche PC, et al. 2011. The Cochrane Collaboration's tool for assessing risk of bias in randomised trials. BMJ 343:d5928.

Higgins JPT, Altman DG. 2008. Assessing risk of bias in included studies. In: Higgins JPT, Green S, eds. Cochrane Handbook for Systematic Reviews of Interventions. Oxford: Wiley; 187-241.

Jares M, Vanek T, Straka Z, Brucek P. 2003. Tranexamic acid reduces bleeding after off-pump coronary artery bypass grafting. J Cardiovasc Surg (Torino) 44:205-8.

Knapik P, Knapik M, Zembala MO, et al. 2019. In-hospital and mid-term outcomes in patients reoperated on due to bleeding following coronary artery surgery (from the KROK Registry). Interact Cardiovasc Thorac Surg, In press.

Kurlansky PA. 2003. Is there a hypercoagulable state after off-pump coronary artery bypass surgery? What do we know and what can we do? J Thorac Cardiovasc Surg 126:7-10.

Laupacis A, Fergusson D. 1997. Drugs to minimize perioperative blood loss in cardiac surgery: Meta-analyses using perioperative blood transfusion as the outcome. The International Study of Peri-operative Transfusion (ISPOT) Investigators. Anesth Analg 85:1258-67.

Mavridis D, Salanti G. 2014. How to assess publication bias: Funnel plot, trim-and-fill method and selection models. Evid Based Ment Health 17:30.

Mehr-Aein A, Sadeghi M, Madani-civi M. 2007. Does tranexamic acid reduce blood loss in off-pump coronary artery bypass? Asian Cardiovasc Thorac Ann 15:285-9.

Moher D, Cook DJ, Eastwood S, Olkin I, Rennie D, Stroup DF. 1999. Improving the quality of reports of meta-analyses of randomised controlled trials: The QUOROM statement. Quality of Reporting of Metaanalyses. Lancet 354:1896-900.

Murphy GJ, Mango E, Lucchetti V, et al. 2006. A randomized trial of tranexamic acid in combination with cell salvage plus a meta-analysis of randomized trials evaluating tranexamic acid in off-pump coronary artery bypass grafting. J Thorac Cardiovasc Surg 132:475-81. 
Myles PS, Smith JA, Forbes A, et al. 2017. Tranexamic acid in patients undergoing coronary-artery surgery. N Engl J Med 376:136-48.

Schulz KF, Grimes DA. 2005. Sample size calculations in randomised trials: Mandatory and mystical. Lancet 365:1348-53.

Shi J, Wang G, Lv H, Yuan S, Wang Y, Ji H, Li L. 2013. Tranexamic acid in on-pump coronary artery bypass grafting without clopidogrel and aspirin cessation: Randomized trial and 1-year follow-up. Ann Thorac Surg 95:795-802.

Taghaddomi RJ, Mirzaee A, Attar AS, Shirdel A. 2009. Tranexamic acid reduces blood loss in off-pump coronary artery bypass surgery. J Cardiothorac Vasc Anesth 23:312-5.

Trevisan D, Zavatti L, Gabbieri D, Pedulli M, Giordano G, Meli M. 2016. Point-of-care-based protocol with first-line therapy with coagulation factor concentrates is associated with decrease allogenic blood transfusion and costs in cardiovascular surgery: An Italian single-center experience. Minerva Anestesiol 82:1077-88.

Vanek T, Jares M, Fajt R, et al. 2005. Fibrinolytic inhibitors in off-pump coronary surgery: A prospective, randomized, double-blind TAP study (tranexamic acid, aprotinin, placebo). Eur J Cardiothorac Surg 28:563-8.

Velioglu Y, Isik M. 2019. Early-term outcomes of off-pump versus onpump beating-heart coronary artery bypass surgery. Thorac Cardiovasc
Surg 67:546-53

Wang G, Xie G, Jiang T, et al. 2012. Tranexamic acid reduces blood loss after off-pump coronary surgery: A prospective, randomized, doubleblind, placebo-controlled study. Anesth Analg 115:239-43.

Wei M, Jian K, Guo Z, et al. 2006. Tranexamic acid reduces postoperative bleeding in off-pump coronary artery bypass grafting. Scand Cardiovasc J 40:105-9.

Xu S, Chen JY, Zheng Q, et al. 2019. The safest and most efficacious route of tranexamic acid administration in total jointarthroplasty: A systematic review and network meta-analysis. Thromb Res 176:61-6.

Zhang Y, Gao X, Yuan S, et al. 2018. Effects of tranexamic acid on shortterm and long-term outcomes of on-pump coronary artery bypass grafting: Randomized trial and 7-year follow-up. Cardiovasc Ther 36:e12472.

Zhou ZF, Zhang FJ, Huo YF, et al. 2017. Intraoperative tranexamic acid is associated with postoperative stroke in patients undergoing cardiac surgery. PLoS One 12:e177011.

Zhou ZF, Jia XP, Sun K, et al. 2017. Mild volume acute normovolemichemodilution is associated with lower intraoperative transfusion and postoperative pulmonary infection in patients undergoing cardiac surgery-A retrospective, propensity matching study. BMC Anesthesiol $17: 13$. 